Artigo Original

Original Article

Vanessa Fernandes de Almeida Porto ${ }^{1}$ (C) Thamires Teles Bezerra ${ }^{1}$ (C)

Fabiana Zambon ${ }^{2,3}$

Mara Behlau² (1D)

Palavras-chaves

Voz

Docentes

Fonoaudiologia

Fadiga Muscular

Autoavaliação

Keywords

Voice

Teachers

Speech Therapy

Muscle Fatigue

Self-Evaluation

Endereço para correspondência:

Vanessa Fernandes de Almeida Porto

Centro de Ciências Integradoras - CCI,

Universidade Estadual de Ciências da

Saúde de Alagoas - UNCISAL

Rua São Domingos, 340, Mangabeiras,

Edifício Aurora, apt. 301, Maceió (AL),

Brasil, CEP: 57037-538.

E-mail: vanessa.porto@uncisal.edu.br

Recebido em: Abril 07, 2020

\section{Fadiga, esforço e desconforto vocal em professores após atividade letiva}

\author{
Fatigue, effort and vocal discomfort in teachers \\ after teaching activity
}

\section{RESUMO}

Objetivo: Investigar a fadiga vocal e sua relação com a sensação de esforço fonatório e desconforto no trato vocal de professores após uma semana de atividade letiva. Método: Estudo transversal, quantitativo, participando 40 professores com queixas de fadiga vocal. Procedimentos realizados no começo e final da semana, antes do início das aulas: Índice de Fadiga Vocal-IFV, Escala Borg, Escala de Desconforto do Trato Vocal-EDTV e registro de voz para análise perceptivo-auditiva. Resultados: Não houve mudanças no esforço fonatório e na frequência e intensidade do desconforto no trato vocal. Em relação ao IFV, nos domínios fadiga e limitação vocal e desconforto físico associado à voz, os professores iniciaram e terminaram a semana com valores compatíveis aos dos disfônicos. No domínio restrição vocal iniciaram a semana com valores compatíveis aos dos indivíduos vocalmente saudáveis e no final da semana tiveram escores compatíveis aos dos disfônicos. Na recuperação com repouso vocal os valores pré e pós foram abaixo da nota de corte, significando menor recuperação vocal. Quanto maior é a sensação de fadiga vocal, maior é a percepção de esforço fonatório; mais frequente é a sensação de aperto, secura, garganta dolorida, sensível e irritada, e mais intensas as sensações de desconforto no trato vocal: aperto, secura, coceira, garganta sensível e irritada. Conclusão: Professores percebem aumento de fadiga vocal, sem mudanças no esforço fonatório e desconforto de trato vocal após uma semana de aula. Quanto maior é a percepção de fadiga vocal, maior é a sensação de esforço e desconforto fonatório.

\begin{abstract}
Purpose: Investigate vocal fatigue and its relationship with the sensation of phonatory effort and discomfort in the vocal tract of teachers after a week of activity. Methods: Cross-sectional, quantitative study, involving 40 teachers with complaints of vocal fatigue. Procedures performed at the beginning and end of the week, before the classes start were Vocal Fatigue Index, Borg Scale, Vocal Tract Discomfort Scale and voice recording for perceptual analysis. Results: There were no changes in phonatory effort and in frequency and intensity of discomfort in vocal tract. In relation to the IFV, in the domains of fatigue and vocal limitation and physical discomfort associated with the voice, teachers started and ended the week with values compatible with dysphonics. In the vocal restriction domain, they started the week with values compatible with vocal healthy individuals and at the end of the week they had scores compatible with dysphonic. In recovery with vocal rest, the pre and post values were below the cut-off score, meaning less vocal recovery. The greater the sensation of vocal fatigue, the greater the perception of phonatory effort; more frequent is the sensation of tightness, dryness, sore, sensitive and irritated throat and more intense the sensations of discomfort in the vocal tract: tightness, dryness, itching, sensitive and irritated throat. Conclusion: Teachers perceive an increase in vocal fatigue, without changes in phonatory effort and vocal tract discomfort after one week of class. The greater the perception of vocal fatigue, the greater the sensation of effort and phonatory discomfort.
\end{abstract}

Trabalho realizado na Universidade Estadual de Ciências da Saúde de Alagoas-UNCISAL, Maceió (AL), Brasil. ${ }^{1}$ Universidade Estadual de Ciências da Saúde de Alagoas - UNCISAL - Maceió (AL), Brasil.

${ }^{2}$ Centro de Estudos da Voz - CEV - São Paulo (SP), Brasil.

${ }^{3}$ Sindicato dos Professores de São Paulo - SinproSP - São Paulo (SP), Brasil.

Financial: Nada a declarar

Conflict of Interest: Nada a declarar 


\section{INTRODUÇÃO}

A voz é uma das principais formas de expressão do ser humano, porém, há um grupo de indivíduos que se destaca pela intensa demanda vocal, denominados profissionais da voz ${ }^{(1)}$.

A voz profissional é definida como "a forma de comunicação oral utilizada por indivíduos que dela dependem para exercer sua atividade ocupacional"(1). Professores utilizam a voz como principal instrumento de trabalho, muitas vezes, em circunstâncias adversas, o que faz com que sejam mais propensos a ter problemas vocais ${ }^{(2)}$.

O uso prolongado da voz associado a fatores de risco individuais, ambientais e de organização do trabalho pode contribuir para elevar a ocorrência de distúrbios vocais em professores $^{(3)}$, sendo o distúrbio de voz uma das principais causas de afastamento do trabalho docente ${ }^{(4)}$. Portanto, estes aspectos podem desencadear ou intensificar os sintomas, tornando essa categoria profissional mais predisposta aos distúrbios vocais ${ }^{(4)}$.

O uso inadequado da voz, frequente entre os professores, muitas vezes é consequência da falta de conhecimento e treinamento ${ }^{(1)}$. Estes profissionais quando expostos a condições inapropriadas de uso da voz ou organização de trabalho desfavorável podem ter que aumentar o esforço vocal ${ }^{(5)}$. Desta forma, muitas vezes observa-se uma associação entre maior carga horária e alteração vocal, com consequente favorecimento de um quadro de fadiga vocal $^{(6)}$. Além disto, o distúrbio de voz relacionado ao trabalho (DVRT) pode ser ocasionado devido a vários fatores provenientes do contexto de trabalho (fatores ambientais, organizacionais, de uso vocal), bem como aos fatores predisponentes do indivíduo ${ }^{(7)}$.

A fadiga vocal é uma percepção que pode se manifestar pelo aumento do esforço fonatório, geralmente associado a um aumento da demanda vocal ${ }^{(8)}$. Além disto, quando associada a comportamentos negativos pode favorecer o fonotrauma e o desenvolvimento de alterações na laringe ${ }^{(8)}$.

Ainda não é claro se a fadiga vocal acontece independente da alteração vocal ou se atua na contribuição desta alteração. Ainda não existe consenso na literatura quanto ao seu conceito e definição universal, podendo se confundir ao conceito de esforço fonatório ${ }^{(9)}$. Um dos primeiros sintomas de que a produção vocal não está sendo saudável, seja por fatores individuais ou como resultado de condições ambientais adversas é a presença de um ou mais sintomas de desconforto no trato vocal, possivelmente resultante de esforço excessivo com relação à fonação ${ }^{(10)}$.

A avaliação da fadiga vocal ainda é um desafio, devido à sua multicausalidade e à falta de instrumentos específicos para isto. Desta forma, acredita-se que um instrumento que mensure a percepção do indivíduo em relação a esse aspecto é importante, principalmente para que se desenvolvam padrões de prevenção e tratamento para alterações vocais adquiridas ${ }^{(11)}$.

Estudos mostram que os sinais e sintomas vocais mais frequentes nos professores possuem pouca relação com a qualidade de voz produzida e estão associados com maior frequência às percepções físicas relacionadas com a produção vocal, tais como fadiga, esforço e desconforto do trato vocal ${ }^{(2,12)}$.

A compreensão das questões relacionadas à percepção de desconforto do trato vocal é imprescindível na avaliação de indivíduos expostos a fatores de risco para o desenvolvimento de um problema de voz, pois, muitas vezes, esses sintomas podem indicar o início de um distúrbio da voz ${ }^{(13)}$ Assim, se faz necessário identificar por meio da pesquisa os diferentes sintomas de distúrbios vocais e promover estratégias de prevenção nessa população.

Desta forma, torna-se importante investigar se há relação entre esforço e sensação de desconforto no trato vocal e a fadiga vocal em professores após uma semana de atividade letiva.

\section{MÉTODO}

O presente estudo foi aprovado pelo Comitê de Ética em Pesquisa da Universidade de Ciências da Saúde de Alagoas - UNCISAL sob o número de CAAE: 11465119.4.0000.501.

\section{Participantes: recrutamento e caracterização}

Inicialmente, os sujeitos foram abordados na sala dos professores e, os que relataram queixa de fadiga vocal foram convidados a participar do estudo. Os participantes receberam informações verbais e por escrito quanto aos objetivos da pesquisa, bem como assinaram o Termo de Consentimento Livre e Esclarecido (TCLE). Em seguida, na sala da coordenação todos os professores responderam aos protocolos, bem como foi realizada o registro das vozes. Todos os dados foram coletados nos meses de setembro e outubro de 2019.

Trata-se de um estudo transversal, quantitativo, em que foram incluídos professores com queixa de fadiga vocal, idade entre 18 e 45 anos, carga horária semanal de $20 \mathrm{~h}$ ( $04 \mathrm{~h}$ por dia), com no mínimo um ano de docência. Foram excluídos os professores de Educação Física, além daqueles que estivessem com algum comprometimento de via aérea superior no dia da coleta, como resfriados ou gripes.

\section{Procedimentos}

O questionário para caracterização da amostra foi desenvolvido para traçar o perfil dos participantes da pesquisa, apresentando questões referentes a disciplinas e séries que lecionam, número médio de alunos por sala, tempo que lecionam, se há ruído na sala ou se já consultou um médico devido a problemas de voz.

O protocolo Índice de Fadiga Vocal (IFV) foi desenvolvido para investigar a sensação de fadiga vocal, sendo utilizada nesta pesquisa a versão validada e adaptada para o português brasileiro que, diferentemente da versão original em inglês ${ }^{(14)}$, apresenta 17 questões divididas em quatro domínios: fadiga e limitação vocal, restrição vocal, desconforto físico associado à voz $\mathrm{e}$ recuperação com repouso vocal. Maiores valores significam aumento dos sintomas, com exceção da recuperação com o repouso vocal, no qual os maiores valores significam maior recuperação vocal ${ }^{(15)}$. O cálculo do escore total é realizado por meio da seguinte fórmula: Total $=$ Fator $1+$ Fator $2+$ Fator $3+$ (12 - Fator 4). Os valores de corte para cada fator são 4,50 para fadiga e limitação vocal (fator 1), 3,50 para restrição vocal (fator 2), 1,50 para desconforto físico associado à voz (fator 3) e 8,50 para recuperação com repouso vocal (fator 4). Para o escore total, o valor que separa os indivíduos disfônicos dos vocalmente saudáveis é de $11,50^{(15)}$. 
Destaca-se que pontuações mais altas para o fator 4 representam melhora dos sintomas de fadiga vocal com repouso, no entanto isso é distinto do que é observado nos demais fatores ${ }^{(15)}$.

A escala Borg mede a percepção individual do esforço a partir da mensuração de uma escala de 0 a $10^{(16)}$. Já a Escala de Desconforto do Trato Vocal (EDTV) foi desenvolvida como uma ferramenta de mensuração da intensidade e da frequência dos sintomas de desconforto do trato vocal, preenchida pelo próprio indivíduo, por meio do uso de descritores qualitativos. Trata-se de uma escala com 08 sintomas e sensações que podem ser sentidos na garganta. O indivíduo assinala a frequência e intensidade com que os sintomas ocorrem ${ }^{(17)}$, em uma escala que varia de 0 a 6 pontos. Para a frequência da sensação/sintoma, a escala varia de (0) para nunca, (1-2) às vezes, (3-5) muitas vezes e (6) sempre, para a intensidade da sensação/sintoma, a escala varia de (0) para nenhuma, (1-3) leve, (4-5) moderada e (6) extrema.

As vozes foram registradas em computador portátil, captada por microfone unidirecional head set, marca Logitech $350^{\circledR}$, disposto lateralmente a três centímetros $(\mathrm{cm})$ da boca do sujeito a fim de evitar interferência do ruído expiratório. Para fins de catalogação e gravação da voz, os registros foram processados nos programas de análise acústica Voxmetria ${ }^{\circledR}$ versão $2.4 \mathrm{~h}$, e para realização da análise perceptivo-auditiva das emissões. Antes do procedimento, ocorreu uma breve explicação sobre a importância de se manter fonação habitual, de forma que a amostra das vozes captadas fossem a mais espontânea possível. Os sujeitos foram solicitados a emitir de forma sustentada a vogal /e/, seguida de contagem de números de 0 a 10. Posteriormente, as vozes foram analisadas por 03 fonoaudiólogos especialistas em voz, que definiram o grau de desvio vocal, por meio de uma escala $0=$ sem desvio, $1=$ desvio leve, $2=$ desvio moderado,
$3=$ desvio intenso. Para análise da confiabilidade intra-avaliador foi utilizado o teste Kappa de Fleiss, repetiram-se 20\% das amostras de voz e utilizou-se a avaliação do fonoaudiólogo com maior confiabilidade (confiabilidade 76,2\%).

\section{Etapas da coleta}

O desenvolvimento da pesquisa consistiu-se em duas etapas conforme a Figura 1.

No começo da semana antes do início da aula (Etapa 1) e após o término da semana, ou seja, após 20 horas de aula (Etapa 2). Esse procedimento foi adotado com o intuito de verificar a frequência e intensidade dos sintomas de fadiga vocal, esforço fonatório e desconforto do trato vocal antes e após uma semana de aula de um mesmo grupo composto por 40 professores.

Etapa 1 - Antes de iniciar a primeira aula da semana o professor respondeu ao questionário para caracterização da amostra na sala da coordenação. Além disto, foi aplicado o Protocolo IFV, a Escala Borg e a EDTV. Posteriormente, a gravação da emissão sustentada da vogal /e/ e contagem dos números de 1 a 10 para posterior análise perceptivo-auditiva da qualidade vocal.

Etapa 2 - Ao final da semana de aula os participantes responderam novamente o protocolo IFV e as escalas Borg e EDTV. Além disto, realizou novamente o registro da emissão sustentada da vogal/e/ e a contagem dos números de 1 a 10 vozes para comparar quanto a qualidade vocal.

\section{Análise Estatística}

Os dados foram analisados de forma descritiva e inferencial. Utilizou-se o software SPSS 25.0. A descrição das variáveis qualitativas nominais foi realizada por meio de frequência

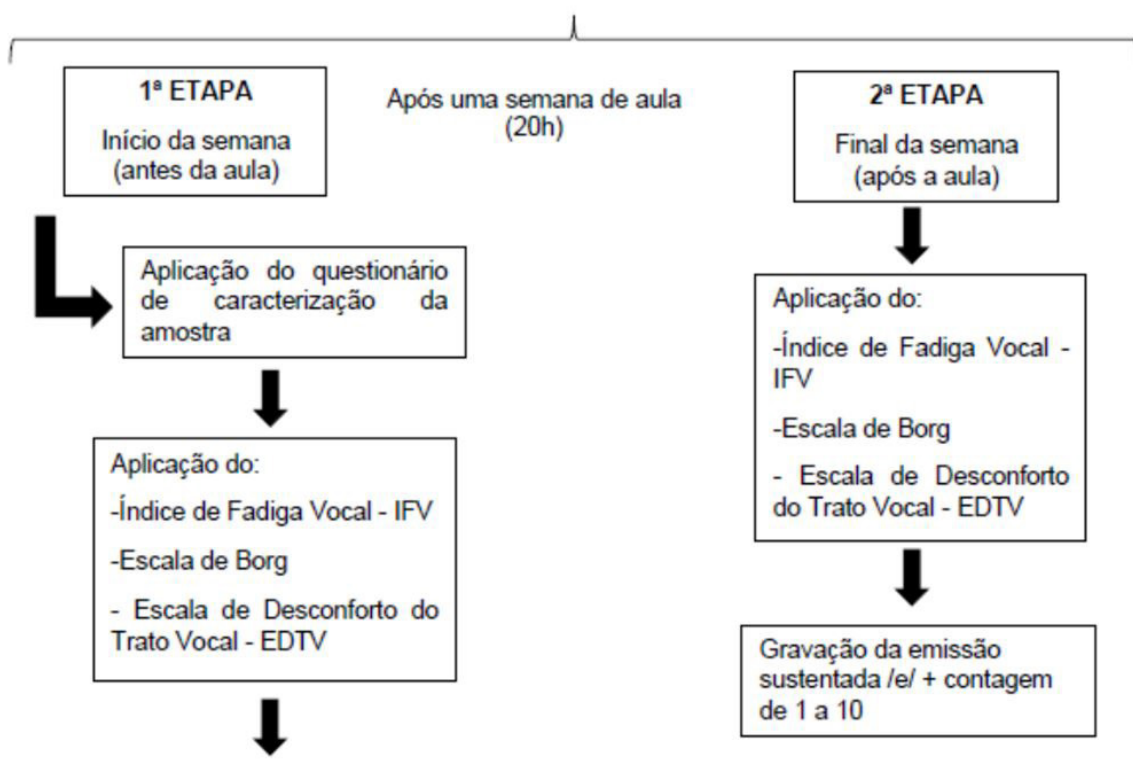

Figura 1. Etapas do desenvolvimento da coleta 
relativa e de frequência absoluta. A descrição das variáveis quantitativas e qualitativas ordinais foi realizada por meio de medidas de variabilidade (desvio-padrão), tendência central (média e mediana) e posição (mínimo, máximo, primeiro quartil e terceiro quartil).

Para realizar a análise inferencial, as variáveis quantitativas passaram por uma análise da homogeneidade de distribuição com o teste Shapiro Wilk. Todos os dados apresentaram distribuição não-normal. Dessa forma, a comparação dos dados qualitativos ordinais e quantitativos não-normais entre os dois grupos dependentes foi realizada com o Teste de Wilcoxon. Considerou-se um nível de significância de 5\% em todas as análises inferenciais. A correlação entre duas variáveis qualitativas ordinais ou quantitativas não-normais foi realizada com o Teste de Correlação de Spearman.

Para verificar a presença de correlação entre os achados foi o utilizado o teste de Correlação de Spearman. Para analisar a força da correlação, foram considerados os valores de $\mathrm{r}=0,10$ até 0,30 como correlação fraca; $r=0,40$ até 0,6 como moderada $e$ $\mathrm{r}=0,70$ até 1 como indicativo de forte correlação ${ }^{(18)}$.

\section{RESULTADOS}

Participaram do presente estudo 40 professores do sexo feminino do Ensino Infantil e Fundamental 1, da rede Pública Municipal de ensino no Município de Maceió, com idade média de 32 anos e 11 meses ( $\mathrm{DP}=7,70)$. O número médio de alunos por sala foi de 17,38 ( $\mathrm{DP}=4,48)$, o tempo médio de magistério foi de 9 anos e cinco meses ( $D P=5,59)$, sendo mais frequentes os professores do nível de ensino infantil (80\%), que eram expostos a ruído $(72,50 \%)$ e que não usavam a voz em excesso fora de sala de aula $(77,50 \%)$.

A Tabela 1 mostra que houve aumento significativo nos escores dos domínios fadiga e limitação vocal $(p=0,004)$, restrição vocal $(p<0,001)$, desconforto físico associado à voz $(\mathrm{p}<0,001)$ e total $(\mathrm{p}=0,004)$ e redução significativa nos escores do fator recuperação com repouso vocal $(\mathrm{p}=0,015)$ após uma semana de atividade letiva em professores.

Comparando-se os dados dos dois momentos da coleta, não houve mudanças na autopercepção de esforço fonatório e da avaliação perceptivo-auditiva antes e após uma semana de atividade letiva em professores.

Comparando-se os dados dos dois momentos da coleta, não houve mudanças na frequência e da intensidade (Tabela 2) do desconforto no trato vocal antes e após uma semana de atividade letiva em professores.

Na Tabela 3 observa-se a correlação da fadiga vocal com o esforço fonatório e o desconforto do trato vocal após atividade letiva em professores. Em relação ao domínio fadiga e limitação vocal houve correlação positiva com o esforço fonatório $(\mathrm{r}=0,435 ; \mathrm{p}=0,005)$. O domínio restrição vocal correlacionouse positivamente com o esforço fonatório $(\mathrm{r}=0,365 ; \mathrm{p}=0,020)$, enquanto que o domínio desconforto físico associado à voz teve correlação positiva com esforço fonatório $(\mathrm{r}=0,355 ; \mathrm{p}=0,025)$. O domínio recuperação com repouso vocal mostrou correlação negativa com a variável frequência de secura $(\mathrm{r}=0,318 ; \mathrm{p}=0,046)$. Já o escore total mostrou correlação positiva com a variável esforço fonatório $(r=0,462 ; \mathrm{p}=0,003)$.

No que se refere à força de correlação, observa-se que os fatores de Fadiga e Limitação Vocal, Restrição vocal e Desconforto físico associado à voz apresentaram algumas correlações de força moderadas. Além disto, o IFV total mostrou correlação moderada com escala de borg, frequência de secura, frequência de garganta sensível, frequência de garganta irritada, frequência total, intensidade de secura, intensidade de garganta sensível, intensidade de garganta irritada, intensidade total. As demais correlações foram fracas e não houve correlação forte.

Tabela 1. Comparação da autopercepção de fadiga vocal, esforço fonatório e avaliação perceptivo-auditiva antes e após atividade letiva em professores

\begin{tabular}{|c|c|c|c|c|c|c|c|c|c|}
\hline Variável & Momento & Média & $\mathrm{DP}$ & Mínimo & Máximo & Q25 & Mediana & Q75 & p-valor \\
\hline \multirow{2}{*}{$\begin{array}{l}\text { IFV Fadiga e limitação } \\
\text { vocal }\end{array}$} & Pré & 11,23 & 6,13 & 0,00 & 24,00 & 6,25 & 11,00 & 15,00 & \multirow[t]{2}{*}{$0,004^{\star}$} \\
\hline & Pós & 13,30 & 6,35 & 0,00 & 26,00 & 8,50 & 13,00 & 18,50 & \\
\hline \multirow[t]{2}{*}{ IFV Restrição vocal } & Pré & 3,40 & 3,02 & 0,00 & 11,00 & 1,00 & 3,00 & 5,00 & \multirow[t]{2}{*}{$<0,001^{*}$} \\
\hline & Pós & 4,73 & 3,05 & 0,00 & 12,00 & 3,00 & 5,00 & 7,00 & \\
\hline \multirow{2}{*}{$\begin{array}{l}\text { IFV Desconforto físico } \\
\text { associado à voz }\end{array}$} & Pré & 3,95 & 3,50 & 0,00 & 13,00 & 1,25 & 3,00 & 6,00 & \multirow[t]{2}{*}{$<0,001^{\prime}$} \\
\hline & Pós & 5,60 & 3,56 & 0,00 & 13,00 & 3,00 & 6,00 & 8,00 & \\
\hline \multirow{2}{*}{$\begin{array}{l}\text { IFV Recuperação com } \\
\text { repouso vocal }\end{array}$} & Pré & 5,28 & 3,88 & 0,00 & 12,00 & 2,00 & 5,00 & 8,00 & \multirow[t]{2}{*}{$0,015^{\star}$} \\
\hline & Pós & 3,83 & 3,43 & 0,00 & 12,00 & 1,00 & 3,00 & 6,00 & \\
\hline \multirow[t]{2}{*}{ IFV Total } & Pré & 23,85 & 10,09 & 6,00 & 49,00 & 17,25 & 22,00 & 28,75 & \multirow[t]{2}{*}{$0,004^{*}$} \\
\hline & Pós & 27,45 & 11,14 & 1,00 & 52,00 & 20,00 & 25,50 & 34,00 & \\
\hline \multirow{2}{*}{$\begin{array}{l}\text { Escala de Borg } \\
\text { (esforço vocal) }\end{array}$} & Pré & 3,49 & 1,73 & 0,00 & 9,00 & 3,00 & 3,00 & 4,00 & \multirow[t]{2}{*}{0,916} \\
\hline & Pós & 3,49 & 2,12 & 0,00 & 9,00 & 2,00 & 3,00 & 4,75 & \\
\hline \multirow{2}{*}{$\begin{array}{l}\text { APA emissão } \\
\text { sustentada }\end{array}$} & Pré & 1,20 & 0,72 & 0,00 & 3,00 & 1,00 & 1,00 & 2,00 & \multirow[t]{2}{*}{0,480} \\
\hline & Pós & 1,15 & 0,70 & 0,00 & 3,00 & 1,00 & 1,00 & 2,00 & \\
\hline \multirow[t]{2}{*}{ APA contagem } & Pré & 0,70 & 0,72 & 0,00 & 2,00 & 0,00 & 1,00 & 1,00 & \multirow[t]{2}{*}{0,480} \\
\hline & Pós & 0,83 & 0,81 & 0,00 & 2,00 & 0,00 & 1,00 & 1,75 & \\
\hline
\end{tabular}

${ }^{*} \mathrm{p}<0,05-$ Teste de Wilcoxon

Legenda: DP = Desvio Padrão; Q25 = Primeiro Quartil; Q75 = Terceiro Quartil; IFV = Índice de Fadiga Vocal; APA = Avaliação Perceptivo-Auditiva 
Tabela 2. Comparação da frequência e intensidade de desconforto no trato vocal antes e após atividade letiva em professores

\begin{tabular}{|c|c|c|c|c|c|c|c|c|c|c|c|c|c|c|c|c|c|}
\hline \multirow{2}{*}{ Sintomas } & \multirow{2}{*}{ Momento } & \multicolumn{2}{|c|}{ Média } & \multicolumn{2}{|c|}{ DP } & \multicolumn{2}{|c|}{ Mínimo } & \multicolumn{2}{|c|}{ Máximo } & \multicolumn{2}{|c|}{ Q25 } & \multicolumn{2}{|c|}{ Mediana } & \multicolumn{2}{|c|}{ Q75 } & \multicolumn{2}{|c|}{ p-valor } \\
\hline & & $\mathrm{F}$ & 1 & $\mathrm{~F}$ & 1 & $\mathrm{~F}$ & 1 & $\mathrm{~F}$ & 1 & $\mathrm{~F}$ & 1 & $\mathrm{~F}$ & 1 & $\mathrm{~F}$ & 1 & $\mathrm{~F}$ & $\mathrm{I}$ \\
\hline \multirow[t]{2}{*}{ Secura } & Pré & 2,98 & 2,75 & 1,94 & 1,68 & 0,00 & 0,00 & 6,00 & 6,00 & 2,00 & 2,00 & 3,00 & 3,00 & 5,00 & 4,00 & 0,91 & 0,28 \\
\hline & Pós & 2,98 & 2,98 & 1,78 & 1,75 & 0,00 & 0,00 & 6,00 & 6,00 & 2,00 & 2,00 & 3,00 & 3,00 & 4,00 & 4,00 & & \\
\hline \multirow{2}{*}{$\begin{array}{c}\text { Garganta } \\
\text { sensível }\end{array}$} & Pré & 1,74 & 1,69 & 1,70 & 1,61 & 0,00 & 0,00 & 5,00 & 5,00 & 0,00 & 0,00 & 2,00 & 1,00 & 3,00 & 3,00 & 0,90 & 0,37 \\
\hline & Pós & 1,70 & 1,90 & 1,68 & 1,84 & 0,00 & 0,00 & 6,00 & 6,00 & 0,00 & 0,00 & 1,00 & 2,00 & 2,00 & 3,75 & & \\
\hline \multirow{2}{*}{$\begin{array}{c}\text { Garganta } \\
\text { irritada }\end{array}$} & Pré & 1,93 & 2,10 & 1,37 & 1,53 & 0,00 & 0,00 & 5,00 & 5,00 & 1,00 & 1,00 & 2,00 & 2,00 & 3,00 & 3,00 & 0,97 & 0,43 \\
\hline & Pós & 1,98 & 2,35 & 1,67 & 1,78 & 0,00 & 0,00 & 6,00 & 6,00 & 1,00 & 1,00 & 2,00 & 2,00 & 3,00 & 4,00 & & \\
\hline \multirow{2}{*}{$\begin{array}{l}\text { Garganta } \\
\text { dolorida }\end{array}$} & Pré & 1,68 & 2,03 & 1,49 & 1,61 & 0,00 & 0,00 & 6,00 & 5,00 & 0,25 & 1,00 & 2,00 & 2,00 & 2,00 & 3,75 & 0,13 & 0,29 \\
\hline & Pós & 2,10 & 2,25 & 1,68 & 1,56 & 0,00 & 0,00 & 6,00 & 6,00 & 1,00 & 1,00 & 2,00 & 2,00 & 3,75 & 4,00 & & \\
\hline \multirow[t]{2}{*}{ Queimação } & Pré & 1,25 & 1,35 & 1,63 & 1,75 & 0,00 & 0,00 & 5,00 & 6,00 & 0,00 & 0,00 & 0,00 & 0,00 & 2,75 & 2,75 & 0,06 & 0,07 \\
\hline & Pós & 1,78 & 1,88 & 1,79 & 1,81 & 0,00 & 0,00 & 6,00 & 6,00 & 0,00 & 0,00 & 2,00 & 2,00 & 3,00 & 3,00 & & \\
\hline \multirow[t]{2}{*}{ Coceira } & Pré & 1,55 & 1,83 & 1,50 & 1,55 & 0,00 & 0,00 & 6,00 & 5,00 & 0,00 & 0,00 & 1,50 & 2,00 & 2,00 & 3,00 & 0,26 & 0,57 \\
\hline & Pós & 1,78 & 1,93 & 1,49 & 1,65 & 0,00 & 0,00 & 6,00 & 6,00 & 0,00 & 0,00 & 2,00 & 2,00 & 3,00 & 3,00 & & \\
\hline \multirow{2}{*}{$\begin{array}{c}\text { Bola na } \\
\text { garganta }\end{array}$} & Pré & 0,93 & 0,98 & 1,37 & 1,42 & 0,00 & 0,00 & 5,00 & 5,00 & 0,00 & 0,00 & 0,00 & 0,00 & 2,00 & 2,00 & 0,30 & 0,12 \\
\hline & Pós & 1,10 & 1,33 & 1,55 & 1,59 & 0,00 & 0,00 & 5,00 & 5,00 & 0,00 & 0,00 & 0,00 & 0,50 & 2,00 & 2,75 & & \\
\hline \multirow[t]{2}{*}{ Aperto } & Pré & 0,98 & 1,15 & 1,37 & 1,56 & 0,00 & 0,00 & 4,00 & 5,00 & 0,00 & 0,00 & 0,00 & 0,00 & 2,00 & 2,00 & 0,08 & 0,42 \\
\hline & Pós & 1,28 & 1,35 & 1,36 & 1,49 & 0,00 & 0,00 & 4,00 & 5,00 & 0,00 & 0,00 & 1,00 & 1,00 & 2,00 & 2,00 & & \\
\hline \multirow[t]{2}{*}{ Total } & Pré & 1,62 & 1,73 & 1,08 & 1,10 & 0,00 & 0,00 & 4,38 & 3,88 & 0,66 & 0,78 & 1,50 & 1,88 & 2,22 & 2,72 & 0,09 & 0,08 \\
\hline & Pós & 1,83 & 1,99 & 1,17 & 1,26 & 0,00 & 0,00 & 4,13 & 5,00 & 0,81 & 0,91 & 1,94 & 1,94 & 2,59 & 3,06 & & \\
\hline
\end{tabular}

${ }^{*} \mathrm{p}<0,05$ - Teste de WilcoxonLegenda: DP = Desvio Padrão; Q25 = Primeiro Quartil; Q75 = Terceiro Quartil; F = Frequência; I= Intensidade

Tabela 3. Correlação da fadiga vocal com o esforço fonatório e o desconforto do trato vocal após atividade letiva em professores

\begin{tabular}{|c|c|c|c|c|c|c|}
\hline & & $\begin{array}{l}\text { IFV Fadiga e } \\
\text { limitação vocal }\end{array}$ & $\begin{array}{c}\text { IFV Restrição } \\
\text { vocal }\end{array}$ & $\begin{array}{c}\text { IFV Desconforto } \\
\text { físico associado } \\
\text { à voz }\end{array}$ & $\begin{array}{c}\text { IFV Recuperação } \\
\text { com repouso } \\
\text { vocal }\end{array}$ & IFV Total \\
\hline \multirow{2}{*}{$\begin{array}{l}\text { Escala de Borg } \\
\text { (esforço vocal) }\end{array}$} & $r$ & 0,435 & 0,365 & 0,355 & 0,058 & 0,462 \\
\hline & p-valor & $0,005^{*}$ & $0,020^{*}$ & $0,025^{\star}$ & 0,724 & $0,003^{\star}$ \\
\hline \multirow{2}{*}{$\begin{array}{l}\text { Frequência } \\
\text { Queimação }\end{array}$} & $r$ & 0,228 & 0,124 & 0,283 & $-0,153$ & 0,226 \\
\hline & $\mathrm{p}$-valor & 0,157 & 0,446 & 0,077 & 0,345 & 0,160 \\
\hline \multirow[t]{2}{*}{ Frequência Aperto } & r & 0,424 & 0,195 & 0,317 & $-0,098$ & 0,387 \\
\hline & $\mathrm{p}$-valor & $0,006^{*}$ & 0,228 & $0,046^{\star}$ & 0,547 & $0,014^{*}$ \\
\hline \multirow[t]{2}{*}{ Frequência Secura } & $r$ & 0,485 & 0,371 & 0,614 & $-0,318$ & 0,476 \\
\hline & p-valor & $0,002^{*}$ & $0,019^{*}$ & $<0,001^{\star}$ & $0,046^{\star}$ & $0,002^{*}$ \\
\hline \multirow{2}{*}{$\begin{array}{l}\text { Frequência } \\
\text { Garganta dolorida }\end{array}$} & $r$ & 0,335 & 0,264 & 0,404 & $-0,286$ & 0,322 \\
\hline & $\mathrm{p}$-valor & $0,034^{*}$ & 0,099 & $0,010^{*}$ & 0,073 & $0,043^{*}$ \\
\hline \multirow{2}{*}{$\begin{array}{l}\text { Frequência } \\
\text { Coceira }\end{array}$} & $r$ & 0,326 & 0,175 & 0,273 & $-0,164$ & 0,309 \\
\hline & $\mathrm{p}$-valor & $0,040^{*}$ & 0,280 & 0,088 & 0,311 & 0,052 \\
\hline \multirow{2}{*}{$\begin{array}{c}\text { Frequência } \\
\text { Garganta sensível }\end{array}$} & $r$ & 0,452 & 0,462 & 0,364 & $-0,109$ & 0,476 \\
\hline & p-valor & $0,003^{*}$ & $0,003^{*}$ & $0,021^{*}$ & 0,502 & $0,002^{*}$ \\
\hline \multirow{2}{*}{$\begin{array}{c}\text { Frequência } \\
\text { Garganta irritada }\end{array}$} & $r$ & 0,47 & 0,507 & 0,521 & $-0,132$ & 0,528 \\
\hline & p-valor & $0,002^{*}$ & $0,001^{*}$ & $0,001^{*}$ & 0,418 & $<0,001^{*}$ \\
\hline \multirow{2}{*}{$\begin{array}{c}\text { Frequência Bola } \\
\text { na garganta }\end{array}$} & $r$ & 0,332 & 0,070 & 0,178 & $-0,066$ & 0,285 \\
\hline & p-valor & $0,036^{*}$ & 0,668 & 0,273 & 0,687 & 0,075 \\
\hline \multirow[t]{2}{*}{ Frequência total } & r & 0,520 & 0,399 & 0,561 & $-0,204$ & 0,534 \\
\hline & p-valor & $0,001^{*}$ & $0,011^{*}$ & $<0,001^{\star}$ & 0,206 & $<0,001^{*}$ \\
\hline \multirow{2}{*}{$\begin{array}{l}\text { Intensidade } \\
\text { Queimação }\end{array}$} & $r$ & 0,317 & 0,185 & 0,353 & $-0,183$ & 0,307 \\
\hline & p-valor & $0,046^{*}$ & 0,253 & $0,026^{*}$ & 0,258 & 0,054 \\
\hline \multirow{2}{*}{$\begin{array}{l}\text { Intensidade } \\
\text { Aperto }\end{array}$} & $r$ & 0,392 & 0,118 & 0,239 & $-0,059$ & 0,337 \\
\hline & $\mathrm{p}$-valor & $0,012^{*}$ & 0,467 & 0,137 & 0,718 & $0,033^{*}$ \\
\hline \multirow{2}{*}{$\begin{array}{l}\text { Intensidade } \\
\text { Secura }\end{array}$} & $r$ & 0,443 & 0,260 & 0,516 & $-0,211$ & 0,434 \\
\hline & $\mathrm{p}$-valor & $0,004^{*}$ & 0,106 & $0,001^{*}$ & 0,191 & $0,005^{\star}$ \\
\hline \multirow{2}{*}{$\begin{array}{l}\text { Intensidade } \\
\text { Garganta dolorida }\end{array}$} & $r$ & 0,286 & 0,183 & 0,328 & $-0,205$ & 0,281 \\
\hline & p-valor & 0,073 & 0,258 & $0,039^{*}$ & 0,205 & 0,079 \\
\hline
\end{tabular}

${ }^{*} \mathrm{p}<0,05$ - Teste de Correlação de Spearman

Legenda: $r$ = Coeficiente de Correlação; IFV= Índice de Fadiga Vocal 
Tabela 3. Continuação...

\begin{tabular}{|c|c|c|c|c|c|c|}
\hline & & $\begin{array}{l}\text { IFV Fadiga e } \\
\text { limitação vocal }\end{array}$ & $\begin{array}{l}\text { IFV Restrição } \\
\text { vocal }\end{array}$ & $\begin{array}{c}\text { IFV Desconforto } \\
\text { físico associado } \\
\text { à voz }\end{array}$ & $\begin{array}{c}\text { IFV Recuperação } \\
\text { com repouso } \\
\text { vocal }\end{array}$ & IFV Tota \\
\hline $\begin{array}{l}\text { Intensidade } \\
\text { Coceira }\end{array}$ & $r$ & 0,417 & 0,186 & 0,329 & $-0,235$ & 0,372 \\
\hline \multirow{2}{*}{$\begin{array}{c}\text { Intensidade } \\
\text { Garganta sensível }\end{array}$} & $r$ & 0,462 & 0,420 & 0,337 & $-0,208$ & 0,445 \\
\hline & $\mathrm{p}$-valor & $0,003^{*}$ & $0,007^{*}$ & $0,034^{*}$ & 0,197 & $0,004^{*}$ \\
\hline $\begin{array}{c}\text { Intensidade } \\
\text { Garganta irritada }\end{array}$ & $r$ & 0,475 & 0,464 & 0,446 & $-0,104$ & 0,509 \\
\hline \multirow{2}{*}{$\begin{array}{l}\text { Intensidade Bola } \\
\text { na garganta }\end{array}$} & r & 0,382 & 0,120 & 0,215 & $-0,113$ & 0,321 \\
\hline & $\mathrm{p}$-valor & $0,015^{\star}$ & 0,461 & 0,182 & 0,486 & $0,044^{*}$ \\
\hline \multirow[t]{2}{*}{ Intensidade total } & $r$ & 0,545 & 0,351 & 0,500 & $-0,170$ & 0,536 \\
\hline & $\mathrm{p}$-valor & $<0,001^{*}$ & $0,026^{*}$ & $0,001^{*}$ & 0,295 & $<0,001^{*}$ \\
\hline
\end{tabular}

${ }^{*} \mathrm{p}<0,05$ - Teste de Correlação de Spearman

Legenda: $r$ = Coeficiente de Correlação; IFV= Índice de Fadiga Vocal

\section{DISCUSSÃO}

A fadiga vocal é uma queixa frequente entre os professores, visto que atuam em ambientes ruidosos, com uma carga horária elevada, condições de trabalho inadequadas e tempo reduzido de repouso vocal ${ }^{(11)}$. Muitos docentes percebem um aumento no esforço fonatório ao longo do tempo, que pode ser acompanhado por uma diminuição da função fonatória ${ }^{(19)}$. Além disto, estes professores apresentam sintomas vocais, que podem variar em frequência e intensidade, comprometendo, em alguns casos, o bem-estar do indivíduo ${ }^{(20)}$.

No que se refere à fadiga vocal, ao comparar-se os resultados do IFV apresentados pelos professores investigados com os valores de corte que separam indivíduos vocalmente saudáveis dos disfônicos da pesquisa de validação do protocolo para o português brasileiro, é possível verificar que nos domínios fadiga e limitação vocal e desconforto físico associado à voz os docentes iniciaram e finalizaram a semana com escores compatíveis aos disfônicos ${ }^{(15)}$. No domínio restrição vocal iniciaram a semana com valores compatíveis aos dos indivíduos vocalmente saudáveis e ao final da semana passaram a apresentar escores compatíveis aos indivíduos disfônicos ${ }^{(15)}$. Em relação ao domínio recuperação com repouso vocal, os valores pré e pós foram abaixo da nota de corte do protocolo IFV, o que mostra que houve menor recuperação vocal, aspecto que pode vir a dificultar o ato de lecionar e colaborar para o desenvolvimento de uma alteração vocal ${ }^{(15)}$. Em relação ao escore total observase que houve aumento no valor total da fadiga vocal após uma semana de atividade letiva ${ }^{(15)}$.

Outro estudo com indivíduos com queixa vocal mostrou resultados distintos, com valores elevados nos domínios fadiga e limitação vocal, restrição vocal e desconforto físico associado à voz ${ }^{(14)}$, dados que não corroboram a presente pesquisa, visto que foram obtidos resultados reduzidos em todos os domínios. Uma pesquisa evidenciou que professores perceberam menor recuperação vocal com o repouso no final do ano do que no início, dado que vai ao encontro do achado no presente estudo. A fadiga vocal do professor, em geral, decorre por consequência de uma sobrecarga da voz em aulas expositivas e a falta de uma recuperação adequada após o uso vocal ${ }^{(19)}$, aspecto que pode limitar e comprometer o uso vocal do docente.

Em relação à percepção do esforço fonatório observa-se que foi moderada e permaneceu a mesma no início e final da semana de atividades letiva. Esse dado corrobora o estudo que investigou indivíduos com hiperfunção vocal após tratamento de quatro semanas e mostrou que o esforço fonatório vocal diminuiu ou não se modificou ${ }^{(20)}$. Este resultado pode ser justificado devido à dificuldade dos pacientes em estimar o esforço vocal produzido no momento da aplicação da escala ou pela ausência de consciência para o monitoramento da sua própria $\operatorname{voZ}^{(20)}$.

Em relação ao desconforto do trato vocal, não foi observada diferença na comparação da frequência e da intensidade antes e após atividade letiva em professores no decorrer da semana. Esse dado se diferencia de estudos que evidenciaram maior frequência e intensidade de sintomas de desconforto no trato vocal de professores após atividade letiva ${ }^{(21,22)}$

Professores referiram muita secura no começo e final da semana. Este dado pode ser justificado pela hidratação insuficiente e o excessivo uso da voz, comportamentos de risco para a saúde vocal ${ }^{(23)}$. Além disto, numa pesquisa com uma população que investigou professores com e sem queixa vocal, foram constatados que os docentes referiam pelo menos três sintomas de desconforto do trato vocal, sendo a secura com maior média, dado que corrobora o presente estudo. Uma pesquisa realizada em São Paulo verificou que docentes que autorreferem a presença de desconforto vocal, identificam a ocorrência igual ou inferior a três sintomas de desconforto, demostrando a sua alta incidência proveniente dessa profissão ${ }^{(10)}$.

Apesar de não haver diferença na comparação pré e pós, observa-se que os professores referem alta frequência e intensidade em vários sintomas de desconforto no trato vocal no decorrer da semana, sendo a secura o mais frequente. Sugere-se que muitos indivíduos disfônicos apresentam algum desconforto no trato vocal, que pode ser decorrente de esforço excessivo, envolvendo a musculatura perilaríngea, por exemplo ${ }^{(10)}$. A presença de sintomas laríngeos, tais como: irritação, secura, pigarro e dor na garganta causam desconfortos que podem interferir na fala, comprometendo a boa produção vocal, o que pode proporcionar perturbações no trabalho, ou no uso vocal social do indivíduo ${ }^{(24)}$. 
Professores do Ensino Fundamental I que apresentam risco vocal referem ao final do dia maior frequência e intensidade dos sintomas de desconforto de trato vocal, sendo que a piora do desconforto pode ter relação com os riscos ocupacionais referentes ao ambiente de trabalho e organização, podendo prejudicar sua saúde vocal ${ }^{(25)}$ Os professores são profissionais que possuem risco vocal moderado ou elevado, dependendo de seus períodos letivos e ambiente de trabalho em que estão inseridos ${ }^{(26)}$.

Quanto maior é a sensação de fadiga vocal, maior é a percepção de esforço fonatório; mais frequente é a sensação de aperto, secura, garganta dolorida, garganta sensível e garganta irritada, e mais intensas são as sensações de desconforto no trato vocal: aperto, secura, coceira, garganta sensível, garganta irritada e bola na garganta. A literatura aponta que professores com queixa vocal apresentam em média 7,8 sintomas, sendo os mais comuns: rouquidão, garganta seca e dor na garganta ${ }^{(10)}$, demonstrando que esses profissionais têm muitos problemas vocais quando comparados a não docentes. Assim, a presença de fadiga vocal aumenta o esforço fonatório e o desconforto no trato vocal, podendo ser fator de risco para o desenvolvimento de uma disfonia nos professores. Nesta perspectiva é importante o docente reconhecer precocemente os sintomas da fadiga, além de condicionar sua voz para retardar o aparecimento dessas manifestações.

Ademais, esse resultado pode ter relação com uma hidratação insuficiente, aspecto que não foi investigado no presente estudo. Ressalta-se que a falta de hidratação pode alterar a viscosidade das pregas vocais e, consequentemente, favorecer a fadiga vocal. Além disto, o limiar de pressão fonatória e o esforço fonatório pode aumentar de acordo com a diminuição da hidratação ${ }^{(27)}$.

Períodos prolongados de fonação acompanhados da falta de hidratação podem levar a alterações na composição dos fluidos das pregas vocais, resultando em elevação da viscosidade e rigidez das dobras. $\mathrm{O}$ aumento da viscosidade do tecido deve resultar em atrito e dissipação de calor proporcionalmente maiores durante a vibração das pregas vocais, consequentemente gerando maior esforço vocal. Essa redução na eficiência fonatória necessita de maior consumo de energia para iniciar e sustentar a oscilação das dobras ${ }^{(27)}$. Sendo assim, é fundamental que os professores sejam orientados sobre a importância de hidratação constante.

A fadiga vocal pode ser influenciada pelo uso excessivo da voz ou aumento da intensidade vocal. Nesse contexto, destaca-se que programas de saúde vocal podem atuar como ferramentas importantes para informar e sensibilizar o professor sobre o bem-estar da voz e colaborar para a diminuição de sintomas e alterações vocais nessa categoria ${ }^{(28)}$. O condicionamento vocal individualizado por meio do aquecimento e desaquecimento da voz é de extrema importância para atender a demanda de uso vocal deste professor com o menor esforço possível. Assim, os professores, que utilizam a voz de forma excessiva, necessitam de orientações básicas sobre a sua produção, seus cuidados e as técnicas vocais ${ }^{(29,30)}$.

Deste modo, medidas educativas de sensibilização em relação ao uso da voz e um treinamento vocal adequado podem contribuir na redução do desconforto e esforço vocal, retardar a fadiga da voz e proporcionar maior resistência vocal. Além disto, podem possibilitar que o professor reconheça precocemente os sintomas vocais e procure uma avaliação médica e fonoaudiológica para um diagnóstico precoce. Desta forma, quanto mais os docentes conhecerem seu instrumento de trabalho, mais poderão conseguir fazer escolhas saudáveis para minimizar o quadro de sintomas vocais e evitar uma alteração vocal.

O estudo apresentou algumas limitações, visto que não realizou a comparação da fadiga vocal entre os professores disfônicos e vocalmente saudáveis. Além disto, não houve controle do ruído da sala em que as vozes foram registradas, bem como a impossibilidade de compreender a fadiga, esforço e desconforto vocal considerando as condições de trabalho e o uso da voz dos professores em situações do seu cotidiano que estejam fora do trabalho na escola. Desta forma, se fazem necessárias futuras pesquisas que contemplem aspectos não estudados no presente estudo.

\section{CONCLUSÕES}

Professores percebem aumento de fadiga vocal, mas não sinalizam mudanças no esforço fonatório e desconforto de trato vocal após uma semana de aula. $\mathrm{O}$ aumento da percepção de fadiga vocal correlaciona-se com o aumento da sensação de esforço e desconforto fonatório.

\section{AGRADECIMENTOS}

Nada a declarar.

\section{REFERENCIAS}

1. Behlau M, Azevedo R, Pontes P. Conceito de voz normal e classificação das disfonias. In: Behlau M. O Livro do especialista. Rio de Janeiro: Revinter; 2001. Vol. 1; p. 64-66.

2. Behlau M, Zambon F, Guerrieri AC, Roy N. Epidemiology of voice disorders in teachers and nonteachers in Brazil: prevalence and adverse effects. J Voice. 2012;26(5):9-18. http://dx.doi.org/10.1016/j.jvoice.2011.09.010. PMid:22516316.

3. Brasil. Ministério da Saúde. Secretaria de Vigilância em Saúde. Departamento de Vigilância em Saúde Ambiental e Saúde do Trabalhador. Distúrbio de Voz Relacionado ao Trabalho - DVRT. Brasília: Ministério da Saúde; 2018.

4. Assunção AA, Oliveira DA. Intensificação do trabalho e saúde dos professores. Educ Soc. 2009;30(107):349-72. http://dx.doi.org/10.1590/ S0101-73302009000200003.

5. Seligmann SE. Desgaste mental no trabalho dominado. Rio de Janeiro: Editora UFRJ; Cortez Editora; 1994. 324 p

6. Ceballos AGC, Carvalho FM, Araujo TM, Reis EJFB. Avaliação perceptivo-auditiva e fatores associados à alteração vocal em professores. Rev Bras Epidemiol. 2011;14(2):285-95. http://dx.doi.org/10.1590/S1415790X2011000200010. PMid:21655695.

7. Freitas CNJ, Almeida AA, Ferreira DAH, Medeiros CMA, Silva MFBL. Condições de trabalho e de voz em professores de escolas públicas e privadas. Audiol Commun Res. 2019;24:21-51. http://dx.doi.org/10.1590/2317-64312019-2151.

8. Zambon F, Moreti F, Nanjundeswaran C, Behlau M. Equivalência cultural da versão brasileira do Vocal Fatigue Index-VFI. CoDAS. 2017;29(2):1-6. http://dx.doi.org/10.1590/2317-1782/20172015261. PMid:28300936. 
9. Welham NV, Maclagan MA. Vocal fatigue: current knowledge and future directions. J Voice. 2003;17(1):21-30. http://dx.doi.org/10.1016/S08921997(03)00033-X. PMid:12705816.

10. Rodrigues G, Zambon F, Mathieson L, Behlau M. Vocal tract discomfort in teachers: its relationship to self-reported voice disorders. J Voice. 2013;27(4):473-80. http://dx.doi.org/10.1016/j.jvoice.2013.01.005. PMid:23528674.

11. Abou Rafée M, Zambon F, Badaro F, Behlau M. Fadiga vocal em professores disfônicos que procuram atendimento fonoaudiológico. CoDAS. 2019;31(3):1-6.

12. Roy N, Merrill RM, Thibeault S, Gray SD, Smith EM. Voice disorders in teachers and the general population: effects on work performance, attendance, and future career choices. J Speech Lang Hear Res. 2004;47(3):542-51. http://dx.doi.org/10.1044/1092-4388(2004/042). PMid:15212567.

13. Tavares EL, Martins RH. Vocal evaluation in teachers with or without symptoms. J Voice. 2007;21(4):407-14. http://dx.doi.org/10.1016/j. jvoice.2006.04.001. PMid:16753282.

14. Nanjundeswaran C, Jacobson BH, Gartner SJ, Abbott KV. Vocal Fatigue Index (VFI): development and validation. J Voice. 2015;29(4):433-20. http://dx.doi.org/10.1016/j.jvoice.2014.09.012. PMid:25795356.

15. Zambon F, Moreti F, Veis VR, Nanjundeswaran C, Behlau M. Vocal Fatigue Index: Validation and Cutoff Values of the Brazilian Version. J Voice. 2020; (In Press). http://dx.doi.org/10.1016/j.jvoice.2020.06.018. PMid:32693976.

16. Baldner EF, Doll E, Vanmersbergen MR. A review of measures of vocal effort with a preliminary study on the establishment of a vocal effort measure. J Voice. 2015;29(5):530-41. http://dx.doi.org/10.1016/j.jvoice.2014.08.017. PMid:26186811.

17. Mathieson L, Hirani SP, Epistein R, Baken RJ, Madeira L, Rubin JS. Laryngeal manual therapy: a preliminary study to examine its treatment effects in the management of muscle tension dysphonia. J Voice. 2009;23(3):35366. http://dx.doi.org/10.1016/j.jvoice.2007.10.002. PMid:18036777.

18. Dancey CP, Reidy J. Estatística sem matemática para psicólogos: usando SPSS para Windows. Porto Alegre: Artmed; 2006. Chapter 6, Análise de correlação: o $r$ de Pearson.

19. Mccabe DJ, Titze IR. Chant therapy for treating vocal fatigue among public school teachers. Am J Speech Lang Pathol. 2002;11(4):356-69. http://dx.doi.org/10.1044/1058-0360(2002/040).

20. Van Leer E, Van Mersbergen M. Using the Borg CR10 physical exertion scale to measure patient-perceived vocal effort pre and post treatment. J Voice. 2017;31(3):19-25.

21. Mendes AF, Lucena BTL, Araujo AMGD, Melo LPF, Lopes LW, Silva MFB. Voz do professor: sintomas de desconforto do trato vocal, intensidade vocal e ruído em sala de aula. CoDAS. 2016;28(2):168-75. http://dx.doi. org/10.1590/2317-1782/20162015027. PMid:27191881.

22. Amaral AC, Zambon F, Moreti F, Behlau M. Desconforto do trato vocal em professores após atividade letiva. CoDAS. 2017;29(2):e20160045. http://dx.doi.org/10.1590/2317-1782/20172016045. PMid:28355385.

23. Ferreira LP, Santos JG, Lima MFB. Sintoma vocal e sua provável causa: levantamento de dados em uma população. Rev CEFAC. 2009;11(1):110-8. http://dx.doi.org/10.1590/S1516-18462009000100015.

24. Menoncin LCM, Jurkievicz AL, Silvério KCA, Camargo PM, Wolf NMN Alterações musculares e esqueléticas cervicais em mulheres disfônicas. Arq Int Otorrinolaringol. 2020;14(4):461-6. http://dx.doi.org/10.1590/ S1809-48722010000400014.

25. Behlau M. Vozes preferidas: considerações sobre opções vocais nas profissões. Fono Atual. 2001;4(16):10-4.

26. Servilha EAM, Ruela IS. Riscos ocupacionais à saúde e voz de professores: especificidades das unidades de rede municipal de ensino. Rev CEFAC. 2010;12(1):109-14. http://dx.doi.org/10.1590/S151618462009005000061 .

27. Welham NV, Maclagan MA. Vocal fatigue: current knowledge and future directions. J Voice. 2003;17(1):21-30. http://dx.doi.org/10.1016/S08921997(03)00033-X. PMid:12705816.

28. Dragone MLS, Ferreira LP, Giannini SPP, Zenari MS, Vieira VP, Behlau M Voz do professor: uma revisão de 15 anos de contribuição fonoaudiológica. Rev Soc Bras Fonoaudiol. 2010;15(2):289-96. http://dx.doi.org/10.1590/ S1516-80342010000200023.

29. Lehto L, Rantala L, Vilkman E, Alku P, Bäckström T. Experiences of a short vocal training course for call-centre customer service advisors. Folia Phoniatr Logop. 2003;55(4):163-76. http://dx.doi.org/10.1159/000071016. PMid:12802089.

30. Ferreira LP, Latorre MRDO, Giannini SPP, Ghirardi ANAM, Karmann DF, Silva EE, et al. Influence of abusive vocal habits, hydration, mastication, and sleep in the occurrence of vocal symptoms in teachers. J Voice. 2010;24(1):86-92. http://dx.doi.org/10.1016/j.jvoice.2008.06.001. PMid:19135852.

\section{Contribuição dos autores}

Todos os autores contribuíram significativamente para o desenvolvimento do artigo. A contribuição de cada autor foi: VFAP foi responsável pela coleta, análise e interpretação dos dados e organização do estudo; FZC foi co-orientadora, responsável pelo delineamento do estudo, análise dos dados e revisão do manuscrito; TTB foi responsável pela coleta de dados e organização do estudo; $M B$ participou, na condição de orientadora, responsável pelo delineamento do estudo e revisão final do manuscrito 